Published in final edited form as:

Obes Manag. 2007 April ; 3(2): 69-73. doi:10.1089/obe.2007.0019.

\title{
Obesity and Polycystic Ovary Syndrome
}

\author{
Susan Sam, MD \\ Assistant Professor of Medicine, Section of Endocrinology, Diabetes and Metabolism University of \\ Illinois Medical Center, Chicago, IL
}

\section{Introduction}

Since its original description in 1935 by Stein and Leventhal, obesity has been recognized as a common feature of the polycystic ovary syndrome (PCOS). In the United States, some studies report that the prevalence of overweight and obesity in women with PCOS is as high as $80 \%$. Outside the U.S., the prevalence of obesity in affected women is lower, although it has increased over time, with studies reporting rates as low as $20 \%$. Differences in the diagnostic criteria for PCOS account for some of this difference in prevalence rates. However, even when comparable diagnostic criteria are applied, both the prevalence and severity of obesity are lower in women with PCOS outside the U.S. This observation suggests that environmental factors, such as lifestyle, contribute to development of obesity in PCOS. The consistent association between PCOS and obesity suggests a biologic basis for this observation. Moreover, obesity exacerbates many of the reproductive and metabolic abnormalities associated with PCOS. This review explores the available data on the mechanisms of this association.

\section{What is PCOS?}

PCOS is the most common hormonal abnormality in reproductive-age women affecting 7\% of this population. The reproductive features of PCOS include increased androgen production and disordered gonadotropin secretion leading to menstrual irregularity, hirsutism, and infertility. ${ }^{1}$ In addition to these important reproductive manifestations, PCOS has metabolic characteristics that include prominent defects in insulin action and $\beta$-cell function, defects that confer a substantially increased risk for glucose intolerance and type 2 diabetes. ${ }^{1,2}$ Obesity is a common finding in women with PCOS and between $40-80 \%$ of women with this condition are reported to be overweight or obese. Familial aggregation of PCOS strongly supports a genetic susceptibility to this disorder. ${ }^{1}$ 


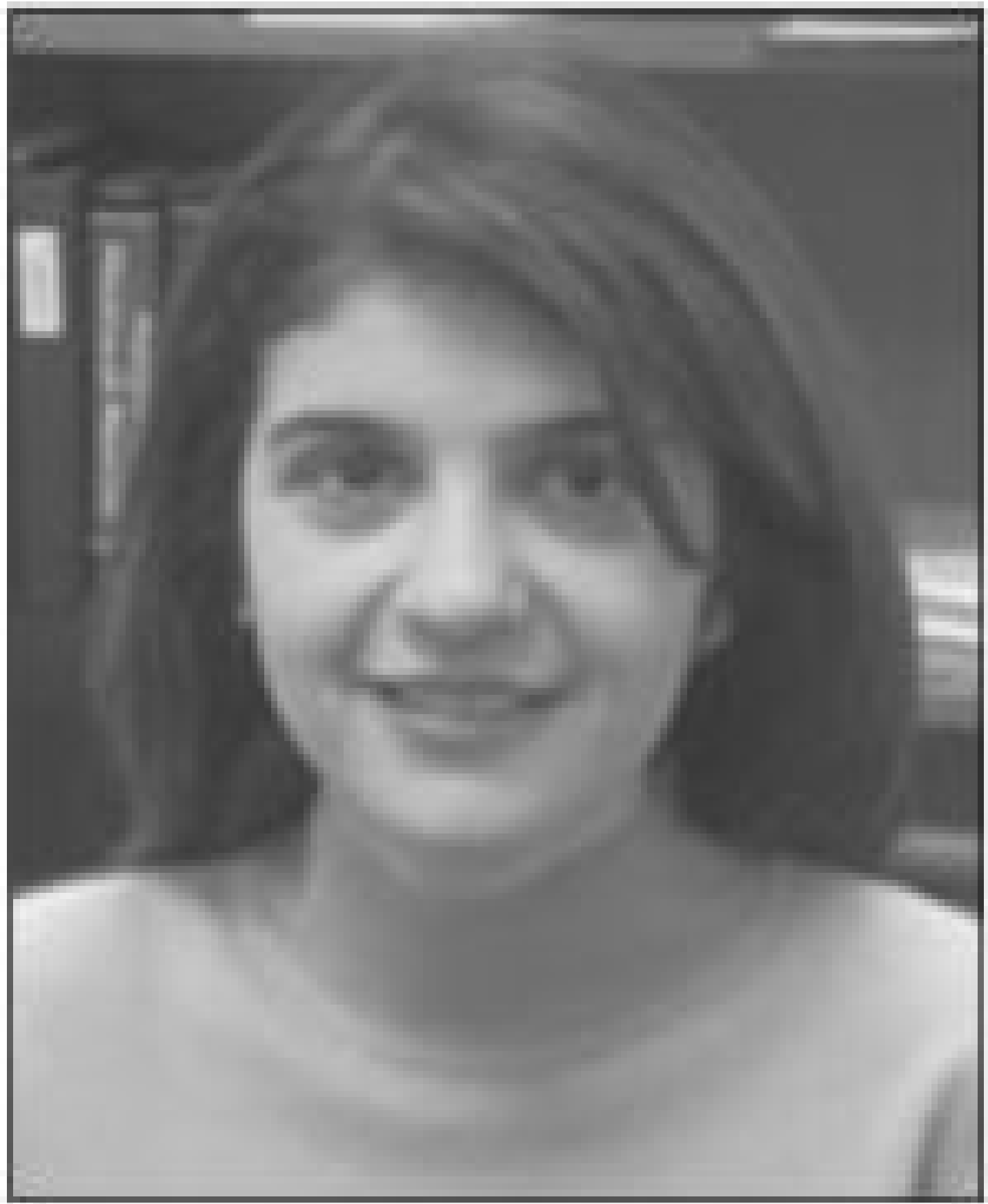

Furthermore, the metabolic abnormalities associated with PCOS, such as $\beta$-cell dysfunction and type 2 diabetes, have heritable components in families of women with PCOS. To date, the genes responsible for PCOS have not been clearly identified. Considering the close association between PCOS and obesity, it is likely that similar or interrelated genes may also predispose to obesity in affected women. No doubt environmental factors (high-caloric diets and reduced exercise) also play a major role in the high prevalence of obesity in women with PCOS.

Until recently, the diagnosis of PCOS was based on the criteria established by a $1990 \mathrm{NIH} /$ National Institutes of Child Health and Human Development (NIH criteria) conference (Table 1). ${ }^{12}$ 
At the 2003 Rotterdam conference on PCOS, the diagnostic criteria were expanded to include polycystic ovary (PCO) morphology (Table 1$).{ }^{3}$ However, this addition to the diagnostic criteria remains controversial because an established minority of women with the biochemical features of the syndrome do not have PCO morphology. Moreover about $25 \%$ of asymptomatic women with regular menses have PCO morphology on ultrasound. Many of these women have elevated androgen or luteinizing hormone (LH) levels, but some have normal reproductive function. Furthermore, ovulatory women with PCO and hyperandrogenism may not be as insulin resistant or carry the same increased risk for type 2 diabetes as women diagnosed with PCOS based on the NIH criteria.

\section{Insulin Action in PCOS: Relation with Obesity}

Insulin resistance is a common finding in PCOS that is independent of obesity. Insulinmediated glucose disposal, reflecting mainly insulin action on skeletal muscle is decreased by $35-40 \%$ in women with PCOS compared to weight comparable reproductively normal women.

2 This defect is independent of but substantially worsened by obesity. In contrast, hepatic insulin resistance, characterized by both increased postabsorptive glucose production and reduced sensitivity to insulin mediated suppression of endogenous glucose production, is present only in obese women with PCOS compared to control women of comparable body weight. ${ }^{2}$ This synergistic deleterious effect of obesity and PCOS on endogenous glucose production may be an important factor in the pathogenesis of glucose intolerance. ${ }^{2}$

Fasting insulin levels are increased in PCOS. Nonetheless, there are defects in insulin secretion that are independent of obesity. ${ }^{2}$ These abnormalities are more pronounced in women with PCOS who have a first-degree relative with type 2 diabetes. In PCOS, basal insulin secretion is increased, but insulin responses to glucose are inappropriately low. ${ }^{2}$ Under normal circumstances, the relation between insulin secretion and sensitivity is constant so that changes in insulin sensitivity are accompanied by reciprocal changes in insulin secretion that maintain normal glucose tolerance; this relationship is known as the "disposition index." Both obese and nonobese women with PCOS have lower a disposition index compared to weight-matched reproductively normal women. ${ }^{2}$ Furthermore, disposition index is significantly lowered by PCOS as well as obesity. In summary, PCOS is associated with defects in insulin sensitivity and secretion that are further exacerbated by obesity.

\section{Glucose Tolerance in PCOS: Relation with Obesity}

Considering the baseline defects in insulin sensitivity and secretion in PCOS and the deleterious impact of obesity on these measures, women with this condition are expected to have a high prevalence of impaired glucose tolerance (IGT, defined by a $2 \mathrm{~h}$ post-challenge glucose level $140-200 \mathrm{mg} / \mathrm{dl}$ ) and type 2 diabetes. A number of studies have confirmed a high prevalence of these abnormalities in obese reproductive-age women with PCOS. In a study of 254 reproductive-age women with PCOS and 80 control women of comparable ethnicity, age, and weight, ${ }^{4}$ the prevalence of glucose intolerance in women with PCOS ( 40\% combined IGT and type 2 diabetes) was much higher than that reported in the control women from the same study (14\% with IGT and $0 \%$ with type 2 diabetes) as well as that reported in a major population-based study (see Figure 1). Furthermore, the risk for developing glucose intolerance increased with increasing body mass index (BMI); the prevalence of IGT and type 2 diabetes were much lower in nonobese women with PCOS (10.3\% and $1.5 \%$, respectively) compared to the obese and the overall population. The study also revealed that normal fasting glucose levels in women with PCOS does not exclude glucose intolerance in these women. Of women diagnosed with type 2 diabetes, $58 \%$ had normal fasting glucose levels and were identified based on elevated $2 \mathrm{~h}$ glucose levels by an oral glucose tolerance test. 
In another study of women with PCOS, ${ }^{5}$ the overall prevalence of glucose intolerance was $45 \%$ (35\% with IGT and 10\% with type 2 diabetes). Women with PCOS and type 2 diabetes were significantly more obese than their counterparts with normal glucose tolerance. Moreover, repeat determination of glucose tolerance after 2.5 years revealed an accelerated rate of conversion from IGT to type 2 diabetes that was strongly dependent upon BMI. Similarly in this study, the fasting glucose levels did not reliably predict the $2 \mathrm{~h}$ glucose levels after a glucose tolerance test. Studies from Australia have also revealed a high prevalence of abnormal glucose tolerance in women with PCOS in association with obesity; ${ }^{6}$ obese women $\left(\mathrm{BMI} \geq 30 \mathrm{~kg} / \mathrm{m}^{2}\right.$ ) had a 10-fold increase and over-weight women (BMI $25-30 \mathrm{~kg} / \mathrm{m}^{2}$ ) had a 7 -fold increase in the risk of abnormal glucose tolerance compared with normal weight $\left(\mathrm{BMI}<25 \mathrm{~kg} / \mathrm{m}^{2}\right.$ ) women with PCOS.

In summary, PCOS is associated with high rates of glucose intolerance resulting from defects in insulin action and $\beta$-cell function. Obesity substantially exacerbates these defects so obese reproductive-age women with PCOS are at very high rates of glucose intolerance. Detection of glucose abnormalities in women with PCOS is best performed by means of glucose tolerance testing, since fasting glucose levels may be normal despite presence of glucose intolerance.

\section{Can Obesity Cause PCOS?}

Reproductive disturbances are more common in obese women regardless of the diagnosis of PCOS. Obese women are more likely to have menstrual irregularity and anvolatory infertility than normal-weight women. In reproductive-age women, the relative risk of anovulatory infertility increases at a BMI of $24 \mathrm{~kg} / \mathrm{m}^{2}$ and continues to rise with increasing BMI. ${ }^{7}$ Consistent with a pathophysiologic role for obesity, weight reduction can restore regular menstrual cycles in these women.

Despite the higher frequency of reproductive abnormalities in obese women, the majority of obese women do not develop hyperandrogenemia and do not have PCOS. In obesity increased androgen production has been reported especially in women with upper-body obesity.

However, androgen clearance rates are also increased, and circulating bioavailable androgens remain in the normal range. In contrast, in PCOS bioavailable androgen levels are increased.

${ }^{1}$ This abnormality is further worsened by obesity, especially central obesity, since sex hormone binding globulin, or SHBG, levels are reduced in this state due to hyperinsulinemia.

Furthermore, PCOS is characterized by abnormalities in the gonadotropin hormone releasing hormone, or $\mathrm{GnRH}$, pulse generator leading to preferential increase in LH release over follicle stimulating hormone (FSH). ${ }^{1}$ These abnormalities are independent of obesity. Moreover, obese reproductively normal women do not have abnormalities in 24-hour LH and FSH plasma concentrations.

Nonetheless, the prevalence of PCOS among obese reproductive-age women has not been well studied. In a recent study from Spain, PCOS was 5-fold more common among unselected premenopausal overweight or obese women seeking advice for weight loss compared to that of the general population ( $28.3 \%$ vs $5.5 \%$, respectively). ${ }^{8}$ In this study, the increased prevalence of PCOS in overweight and obese women was irrespective of the degree of obesity and was independent of the presence or absence of the metabolic syndrome or its features.8 The study demonstrates the prevalence of PCOS may be markedly increased in overweight and obese women. Routine screening by obtaining at least a menstrual history and a careful evaluation for hyperandrogenism may be indicated in these women as well.

\section{Does PCOS Cause Obesity?}

Androgens play an important role in determination of body composition. Men have less body fat with greater distribution of fat in the upper portion of the body (android) compared to 
women, who tend to accumulate fat in the lower portion of the body (gynoid). Vague first reported that the prevalence of diabetes, hypertension, and atherosclerosis was higher in women with android obesity compared to gynoid obesity. ${ }^{9}$ Moreover, he observed that the prevalence of android body habitus increases in women after the age of menopause and women with android obesity tend to have features of hyperandrogenism such as hirsutism.9 Women with upper-body obesity have also been noted to have decreased insulin sensitivity and are at higher risk for cardiovascular disease and diabetes. Independent of BMI, women with PCOS have been reported to have a high prevalence of upper-body obesity as demonstrated by increased waist circumference and waist-hip ratio compared to BMI-matched control women. Consistent with these findings, studies using dual-energy X-ray absorptiometry have revealed increased accumulation of central fat in women with PCOS.10

Chronic exposure to higher testosterone levels in women with PCOS may modify body fat distribution in these women. Support for this hypothesis is provided by studies of androgen administration in nonobese female to male transsexuals that lead to increases in visceral fat and adversely impact insulin sensitivity. ${ }^{11}$ In post-menopausal women exposure to androgens increases visceral fat in both obese and normal-weight women. ${ }^{12}$ In rats, testosterone administration of a single high dose early in life leads to development of insulin resistance and centralization of adipose tissue mass as an adult. ${ }^{13}$ It may be that early androgen exposure adversely impacts future body fat distribution with greater accumulation of central fat.

However, few studies have examined visceral fat content in women with PCOS. Studies of isolated abdominal fat cells from women with PCOS have revealed larger-sized cells in both obese and nonobese women with PCOS compared to control women, suggesting a preferential abdominal accumulation of adipose tissue. ${ }^{14}$ Femoral adipocytes are smaller in obese women with PCOS than reproductively normal women consistent with a shift to android body fat distribution in PCOS women. These observations raise the hypothesis that hyperandrogenemia may contribute to the development of visceral adiposity in PCOS women necessitating further investigation in this area.

\section{Hormonal Regulations of Weight and Appetite}

Lower fasting levels of the peptide hormone, ghrelin, have been reported in women with PCOS compared to weight-matched control women. Ghrelin is produced by the gastric endocrine cells and has been implicated in regulation of appetite and body weight. Ghrelin levels increase sharply before meals leading to hunger and initiation of food intake and drop after feeding leading to suppression of appetite and satiety. Fasting ghrelin levels are reported to be lower in obese individuals due to chronic positive energy balance. However, there is evidence that ghrelin homeostasis in PCOS may be dysregulated. In addition to lower fasting ghrelin levels, women with PCOS have less marked post-parandial reduction in the level of this hormone, as well as less satiety following a test meal. Lack of suppression of ghrelin following food intake may interfere with meal termination and lead to weight gain in these women. ${ }^{15}$

\section{Conclusions}

Obesity is a common finding in PCOS and aggravates many of its reproductive and metabolic features. The relationship between PCOS and obesity is complex, not well understood, and most likely involves interaction of genetic and environmental factors.

\section{References}

1. Sam S, Dunaif A. Polycystic ovary syndrome: syndrome XX? Trends Endocrinol Metab 2003;14:365370. [PubMed: 14516934] 
2. Dunaif A. Insulin action in the polycystic ovary syndrome. Endocrinol Metab Clin North Am 1999;28:341-359. [PubMed: 10352922]

3. Revised 2003 consensus on diagnostic criteria and long-term health risks related to polycystic ovary syndrome. Fertil Steril 2004;81:19-25.

4. Legro RS, Kunselman AR, Dodson WC, Dunaif A. Prevalence and predictors of risk for type 2 diabetes mellitus and impaired glucose tolerance in polycystic ovary syndrome: a prospective, controlled study in 254 affected women. J Clin Endocrinol Metab 1999;84:165-169. [PubMed: 9920077]

5. Ehrmann DA, Barnes RB, Rosenfield RL, Cavaghan MK, Imperial J. Prevalence of impaired glucose tolerance and diabetes in women with polycystic ovary syndrome. Diabetes Care 1999;22:141-146. [PubMed: 10333916]

6. Norman RJ, Masters L, Milner CR, Wang JX, Davies MJ. Relative risk of conversion from normoglycaemia to impaired glucose tolerance or non-insulin dependent diabetes mellitus in polycystic ovarian syndrome. Hum Reprod 2001;16:1995-1998. [PubMed: 11527911]

7. Rich-Edwards JW, Spiegelman D, Garland M, et al. Physical activity, body mass index, and ovulatory disorder infertility. Epidemiology 2002;13:184-190. [PubMed: 11880759]

8. Alvarez-Blasco F, Botella-Carretero JI, San Millan JL, Escobar-Morreale HF. Prevalence and characteristics of the polycystic ovary syndrome in overweight and obese women. Arch Intern Med 2006;166:2081-2086. [PubMed: 17060537]

9. Vague J. The degree of masculine differentiation of obesities: A factor determining predisposition to diabetes, atherosclerosis, gout, and uric calculous disease. Am J Clin Nutr 1956;4:20-34. [PubMed: 13282851]

10. Douchi T, Ijuin H, Nakamura S, Oki T, Yamamoto S, Nagata Y. Body fat distribution in women with polycystic ovary syndrome. Obstet Gynecol 1995;86:516-519. [PubMed: 7675372]

11. Elbers JM, Asscheman H, Seidell JC, Megens JA, Gooren LJ. Long-term testosterone administration increases visceral fat in female to male transsexuals. J Clin Endocrinol Metab 1997;82:2044-2047. [PubMed: 9215270]

12. Rosenfield RL. Ovarian and adrenal function in polycystic ovary syndrome. Endocrinol Metab Clin North Am 1999;28:265-293. [PubMed: 10352919]

13. Nilsson C, Niklasson M, Eriksson E, Bjorntorp P, Holmang A. Imprinting of female offspring with testosterone results in insulin resistance and changes in body fat distribution at adult age in rats. $\mathrm{J}$ Clin Invest 1998;101:74-78. [PubMed: 9421468]

14. Dunaif A, Segal KR, Shelley DR, Green G, Dobrjansky A, Licholai T. Evidence for distinctive and intrinsic defects in insulin action in polycystic ovary syndrome. Diabetes 1992;41:1257-1266. [PubMed: 1397698]

15. Moran LJ, Noakes M, Clifton PM, et al. Ghrelin and measures of satiety are altered in polycystic ovary syndrome but not differentially affected by diet composition. J Clin Endocrinol Metab 2004;89:3337-3344. [PubMed: 15240612] 


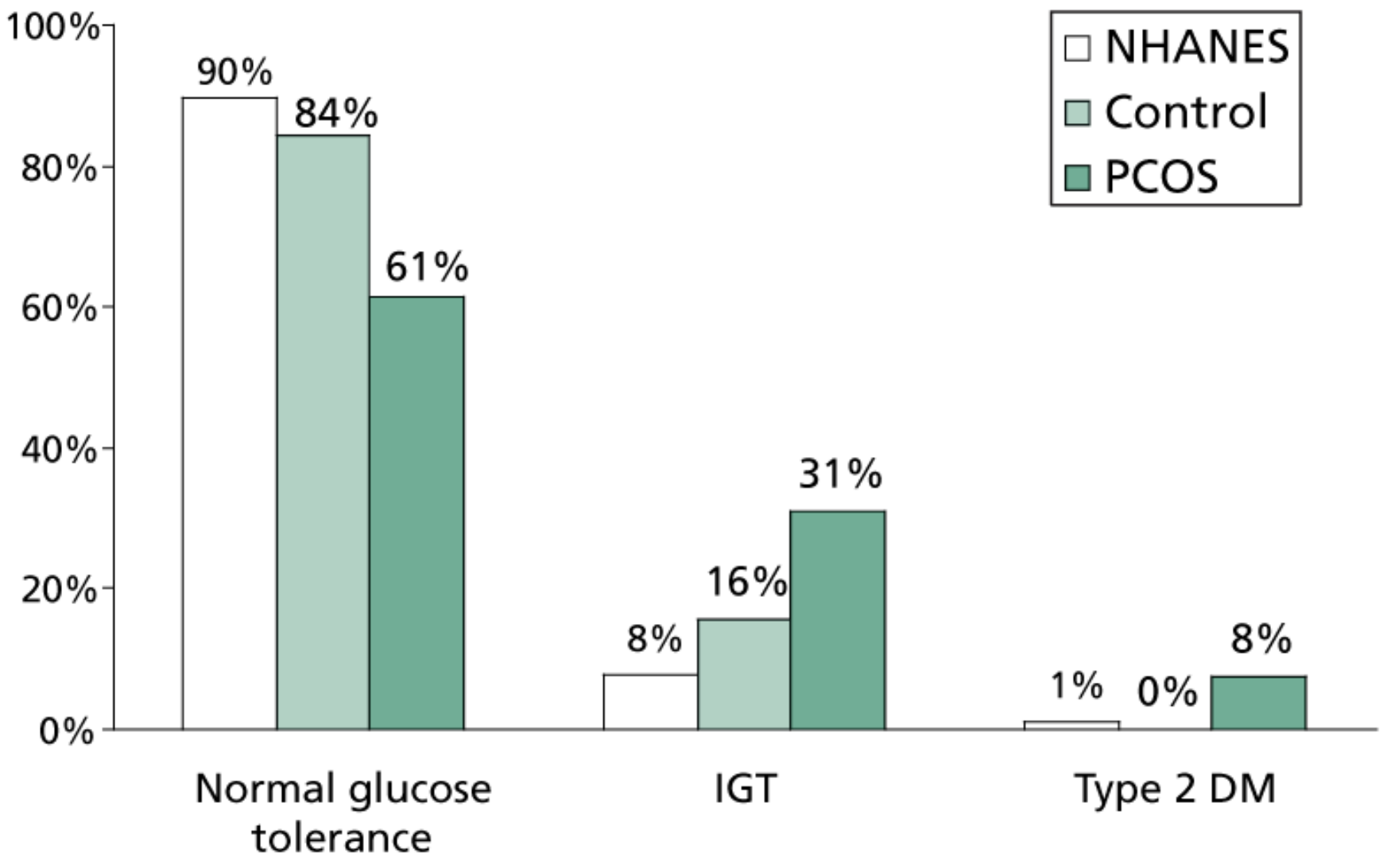

Figure 1.

Women with PCOS (black bars) had much higher prevalence of abnormal glucose tolerance compared to control women of similar ethnicity, age, and weight (gray bars) $(\mathrm{P}=0.02)$ as well as compared to reproductive-age women from the Second National Health and Nutrition Examination Survey (NHANES) (white bars). 4 
Table 1

\section{Diagnostic Criteria for PCOS}

\begin{tabular}{|ll|}
\hline NIH/NICHD Criteria & Rotterdam Criteria $^{*}$ \\
\hline Diagnosis requires both features: & Diagnosis requires 2 of 3 features: \\
\hline 1. Oligo and/or anovulation & 1. Oligo and/or anovulation \\
\hline $\begin{array}{l}\text { 2. Hyperandrogenism } \\
\text { Clinical or biochemical }\end{array}$ & $\begin{array}{l}\text { 2. Hyperandrogenism } \\
\text { Clinical or biochemical }\end{array}$ \\
\hline & 3. Polycystic ovary morphology \\
\end{tabular}

Other androgen excess or related disorders have to be excluded prior to diagnosis of PCOS.

**

Defined by at least one ovary demonstrating an ovarian volume $>10 \mathrm{ml}$ or presence of 12 or more follicles measuring $2-9 \mathrm{~mm}$ in size. 